\title{
Verhaltensökonomik als Gegenprogramm zur Standardökonomik?: Kommentar zum Beitrag von Dennis Dittrich
}

\author{
Christian Schubert
}

Online publiziert: 25. Februar 2019

(C) List-Gesellschaft e.V. 2019

In seinem anregenden und lesenswerten Beitrag zeichnet Dennis Dittrich das Bild einer psychologisch informierten Ökonomik (der unglückliche Begriff ,,Verhaltensökonomik“" hat sich durchgesetzt), die sich in den vergangenen 20 Jahren auf breiter Front etabliert hat und im Begriff steht, sich zum neuen ökonomischen „Mainstream“ zu mausern. Dittrich spricht von einer „Konvergenz von Verhaltensökonomik und ... Standardökonomik".

Im Prinzip ist diesem Befund zuzustimmen. Ein Blick in die aktuellen Ausgaben der ökonomischen Top-Journals genügt, um zu erkennen, dass sich die Ökonomik psychologischen Erkenntnissen geöffnet hat und einst geringgeschätzte Methoden wie insbesondere Experimente inzwischen aus der Spitzenforschung nicht mehr wegzudenken sind. Indes gibt es ein paar Details, die in dieser großen Konvergenzerzählung zuweilen aus dem Blick geraten. Ich werde mich zunächst diesen Details zuwenden, um sodann im zweiten Teil meines Kommentars ein paar Anmerkungen bezüglich der „Politikimplikationen“ verhaltensökonomischer Erkenntnisse zu machen.

\section{1 „Konvergenz“?}

Unbestritten ist, dass wir es in der Ökonomik inzwischen mit einem „,neuen Mainstream“ zu tun haben, der bereitwillig psychologische Erkenntnisse aufgreift und versucht, sie in seine Modelle einzubauen. Insofern hat sich die Verhaltensökono-

This commentary article refers to the article available online at https://doi.org/10.1007/s41025-01900141-8.

C. Schubert $(\bowtie)$

Dep. of Economics, German University in Cairo, Kairo, Ägypten

E-Mail: dr.c.schubert@gmail.com 
mik quasi wie Zucker im Kaffee der Standardökonomik aufgelöst und damit auch diese Standardökonomik verändert. Soweit, so gut. ${ }^{1}$

Es gibt indes aus zwei völlig unterschiedlichen Ecken nachhaltig Kritik an dieser Entwicklung. Zum einen von traditionellen neoklassischen Ökonomen - zu nennen sind hier natürlich Gul und Pesendorfer (2008), deren scharfe Einwände z. B. gegen die Übernahme psychologischer oder neurowissenschaftlicher Methoden in die Ökonomik bei Dittrich unerwähnt bleiben. Nun kann man bezüglich der Qualität dieser Einwände streiten (z. B. Hands 2013) oder auch darüber, wie „repräsentativ“ Gul und Pesendorfer für die Zunft der Ökonomen sind, aber nach den Erfahrungen dieses Kommentators teilen z. B. im deutschen Sprachraum viele zumal ältere Kollegen ihre Einwände.

Der zweite fundamentale Einwand kommt aus der Ecke der Psychologie: Berg und Gigerenzer (2010) etwa bemängeln an dem - sich schon 2010 abzeichnenden „,neuen Mainstream“, er versuche lediglich, den Fit seiner Modelle mit empirischen Daten zu maximieren und verliere dabei das Ziel aus den Augen, das doch darin bestehen solle, die mentalen Prozesse zu erklären, welche beobachtetem Verhalten zugrunde liegen. Dittrich erwähnt die damit eng zusammenhängende Kritik Werner Güths (,neoklassischer Reparaturbetrieb“), scheint diese Art von Einwänden und ihre Implikate aber nicht recht ernst zu nehmen.

Ein weiteres (drittes) Problem sei nur kurz erwähnt; es bewirkt wie das metaphorische Steinchen im Schuh, dass traditionelle Ökonomen ein diffuses Unbehagen beim Studium verhaltensökonomischer Literatur - und insbesondere auch verhaltensökonomischer Politikempfehlungen (dazu sogleich mehr) beschleicht: Wie Dittrich beiläufig erwähnt, fokussiert die moderne Verhaltensökonomik auf das einzelne Individuum und die Qualität seiner Entscheidungen. Notorisch unterbelichtet bleibt dabei die Ebene der institutionellen Arrangements, innerhalb derer es sich bewegt (Ross 2014) - die potentiell rationalitätsfördernde Rolle von Märkten etwa wird zwar von Experimentalökonomen der Vernon Smith'schen Denkrichtung betont, aber im Mainstream der neuen Verhaltensökonomik spielt sie eine untergeordnete Rolle. Der frühe Beitrag von Frey und Eichenberger (1989) zur komplexen Interaktion von monetären Anreizen und „Anomalien“ fand kaum ein Echo in der „neuen“ Verhaltensökonomik.

Ein viertes Problem schließlich steht der harmonischen Integration psychologischer Erkenntnisse im Wege. In den letzten Jahren ist die Psychologie von einer umfassenden Replikationskrise heimgesucht worden, die naturgemäß auch die Verhaltensökonomik betrifft. Nur als Beispiel seien die jüngeren Studien von Yechiam (2018) und Gal und Rucker (2018) erwähnt, die Zweifel an dem Konzept der Verlustaversion wecken, immerhin einem der prominentesten Beiträge der Verhaltensökonomik. Derlei Debatten irritieren Ökonomen, die in axiomatisch fundierter Neo-

\footnotetext{
1 Dittrichs Charakterisierung der Standardökonomik indes ist fragwürdig (S. 11): Sie sei u. a. geprägt vom methodologischen Instrumentalismus. Wenn damit der auf Friedman zurückgehende einseitige Fokus auf korrekte Vorhersagen gemeint sein sollte: Der einseitige Fokus auf der Qualität von Vorhersagen (statt von Erklärungen) sowie die Abwertung der Frage nach dem ,Realismus“ der Modellannahmen steht einer erfolgreichen Integration der Verhaltensökonomik eher entgegen, als sie zu stützen. Ich bezweifle aber ohnehin, dass die meisten Standardökonomen - interessierten sie sich denn für derlei methodologische Fragen - sich als Instrumentalisten verstehen würden (Schubert 2015b).
} 
klassik sozialisiert wurden. Wahr ist aber auch: Die Bedeutung dieser methodologischen Krise wird häufig übertrieben dargestellt, sollte man sie doch eher als Indiz einer normal im trial-and-error-Modus fortschreitenden empirischen Wissenschaft ansehen denn als Sargnagel für die Verhaltensökonomik. Aber in einer Würdigung des derzeitigen Standes der Integration psychologischer Erkenntnisse in die Ökonomik sollte sie dennoch nicht unerwähnt bleiben.

\section{Verhaltensökonomik und Politik}

Dittrichs Ausführungen zu möglichen Politikimplikationen verhaltensökonomischer Erkenntnisse sind zum Teil ergänzungsbedürftig. Vorab: Eine umfassende Diskussion möglicher Politikimplikationen sollte auch eine spezifische Anwendung berücksichtigen, die bei Dittrich unerwähnt bleibt: Die Behavioral Political Economy versucht, aufbauend auf der Public Choice-Forschung politische Prozesse mit den Mitteln der Verhaltensökonomik zu erklären (z. B. Schnellenbach und Schubert 2015). Das Forschungsfeld ist noch jung - wobei zu konzedieren ist, dass Abweichungen vom Homo Oeconomicus-Konzept auch in der traditionellen ökonomischen Analyse von Politik eine Rolle spielten, wenn es z.B. darum ging, die Teilnahme an Wahlen zu erklären: Wenn Millionen andere Menschen ebenfalls wählen gehen, dann kann ich meinen eigenen (kostenträchtigen) Gang zum Wahllokal nicht mehr instrumentell rational erklären, sondern muss die Nutzenfunktion um z.B. Aspekte intrinsischen oder ,expressiven“ Nutzens ergänzen.

Was die eigentliche Frage der Politikimplikationen betrifft, so konzentriere ich mich aus Raumgründen auf einen besonders wichtigen Aspekt, der bei den Versuchen, eine Brücke von rein positiven Erkenntnissen zu normativen Aussagen zu schlagen (ohne dabei dem naturalistischen Fehlschluss zu erliegen), eine zentrale Rolle spielt, nämlich den Ansatz, die real vorgefundenen Präferenzen der Individuen von all ihren Inkonsistenzen zu ,säubern“, um sodann auf Grundlage der im Lichte vollkommener Widerspruchsfreiheit erstrahlenden „sauberen“ Präferenzen Wohlfahrtseffekte zu kalkulieren. Dieses nicht nur auf den ersten Blick befremdlich wirkende Vorgehen erfreut sich im Mainstream der „,neuen“ Verhaltensökonomik großer Beliebtheit; man kann es als Standardansatz der „Behavioral Welfare Economics" bezeichnen, deren wichtigste Vertreter Dittrich kurz erwähnt. Mehr oder weniger explizit liegt dieses Vorgehen auch den momentan so populären „Nudge“Ansätzen zugrunde. ${ }^{2}$ Dort geht es darum, irrationale Entscheider dazu zu bewegen, ihren vermeintlich ,wahren“ Präferenzen gemäß zu handeln.

Dieser „pragmatische“, technokratischen Lenkungszwecken so offenkundig entgegenkommende Ansatz ist nicht nur scharfer philosophischer Kritik ausgesetzt worden (z. B. Hausman und Welch 2010) - die z.B. auch Zweifel weckt, inwiefern Nudging die „Entscheidungssouveränität“ des Individuums respektiert, wie Dittrich kühn behauptet (S. 9) - sondern auch aus Sicht einer eher kontraktualistisch ausge-

\footnotetext{
2 Das Nudge-Instrumentarium geht zurück auf einen kurzen Aufsatz von Thaler und Sunstein (2003). Zur weithin vernachlässigten politökonomischen Dimension des Nudging vgl. Schubert (2017) und Schnellenbach (2012).
} 
richteten Verhaltensökonomik, wo das ganze Konzept, die (kontrafaktischen) gereinigten als die ,wahren“ Präferenzen auszuzeichnen, in Frage gestellt wird (Infante et al. 2016).

Die Frage, wie Wohlfahrt in einer verhaltensökonomischen Welt zu denken ist, ist derzeit noch vollkommen offen. Der verhaltensökonomische Mainstream - wie Dittrich richtig bemerkt, am besten repräsentiert durch Chetty (2015) - macht es sich einfach und setzt Wohlfahrt entweder - wie Chetty selbst - nonchalant mit subjektivem Wohlbefinden (vulgo: Happiness) gleich, oder definiert es eben als Grad der Befriedigung adäquat gesäuberter Präferenzen. Beides ist aus Sicht einer Ökonomik, die psychologisch, aber auch philosophisch ernstgenommen werden will, hochgradig problematisch. ${ }^{3}$ Die sich an der Schnittstelle zwischen Ökonomik, Psychologie und Philosophie anschließende Debatte dreht sich momentan z. B. um folgende Fragen: Existieren „wahre“ Präferenzen (Fumagalli 2013)? Ist Rationalität, standardmäßig verstanden als Konsistenz, überhaupt ein erstrebenswertes Ziel (Berg 2014)? Sollte statt der Befriedigung gegebener Präferenzen eher das kontinuierliche Lernen neuer Präferenzen in den Wohlfahrtsbegriff einfließen (Schubert 2015a)? All das zeigt: Nudges sind nicht das letzte Wort, wenn es darum geht, nach den Politikimplikationen moderner Verhaltensökonomik zu fragen.

\section{Literatur}

Berg, N. (2014). The consistency and ecological rationality approaches to normative bounded rationality. Journal of Economic Methodology, 21, 375-395.

Berg, N., Gigerenzer, G. (2010). As-if behavioral economics: Neoclassical economics in disguise? History of Economic Ideas, 18(1):133-165.

Chetty, R. (2015). Behavioral Economics and public policy: A pragmatist perspective. American Economic Review, 105, 1-33.

Frey, B.S., \& Eichenberger, R. (1989). Economic incentives transform psychological anomalies. Journal of Economic Behavior and Organization, 23, 215-234.

Frey, B. S., \& Stutzer, A. (2012). The use of happiness research for public policy. Social Choice \& Welfare, 38, 659-674.

Fumagalli, R. (2013). The futile search for true utility. Economics and Philosophy, 29, 325-347.

Gal, D., \& Rucker, D. (2018). The loss of loss aversion: Will it loom larger than its gain? Journal of Consumer Psychology, 28, 497-516.

Gul, F., \& Pesendorfer, W. (2008). The case for mindless economics. In A. Caplin \& A. Sullivan (Hrsg.), The foundations of positive and normative economics. Oxford: Oxford University Press.

Hands, D. W. (2013). GP08 is the new F53: Gul and Pesendorfer's methodological essay from the viewpoint of Blaug's Popperian methodology. In M. Boumans \& M. Klaes (Hrsg.), M. Blaug: Rebel with many causes (S. 245-266). Cheltenham: Edward Elgar.

Hausman, D. M., Welch, B. (2010). Debate: To nudge or not to nudge? Journal of Political Philosophy, 18:123-136.

Infante, G., Lecouteux, G., \& Sugden, R. (2016). Preference purification and the inner rational agent: A critique of the conventional wisdom of behavioural welfare economics. Journal of Economic Methodology, 23, 1-25.

Ross, D. (2014). Philosophy of economics. London: Palgrave.

Schnellenbach, J. (2012). Nudges and norms: On the political economy of libertarian paternalism. European Journal of Political Economy, 28, 266-277.

Schnellenbach, J., \& Schubert, C. (2015). Behavioral political economy: A survey. European Journal of Political Economy, 40, 395-417.

Schubert, C. (2015a). Opportunity and preference learning. Economics and Philosophy, 31, 275-295.

\footnotetext{
3 Zu den Problemen, Happiness als Maximand zu nutzen, vgl. nur Frey und Stutzer (2012).
} 
Schubert, C. (2015b). Behavioral Economics: Anmerkungen zum gleichnamigen Buch von Hanno Beck. Ordo, 66(1), 428-436. https://doi.org/10.1515/9783110506044-039.

Schubert, C. (2017). Exploring the (behavioural) political economy of nudging. Journal of Institutional Economics, 13, 499-522.

Thaler, R. H., \& Sunstein, C. R. (2003). Libertarian paternalism. American Economic Review, 93, 175-179. Yechiam, E. (2018). Acceptable losses: The debatable origins of loss aversion. Psychological Research. https://doi.org/10.1007/s00426-018-1013-8. 